\title{
Gravitational Lenses as Long-Base-Line Gravitational-Wave Detectors
}

\author{
B. Allen \\ Department of Physics and Astronomy, Tufts University, Medford, Massachusetts 02155 \\ and Department of Physics, University of Wisconsin at Milwaukee, P.O. Box 413, Milwaukee, Wisconsin 53201
}

(Received 14 August 1989; revised manuscript received 8 September 1989)

\begin{abstract}
Gravitational waves produce a time delay between the different images of a gravitational lens. The measurements of the time delay in the gravitational lens $0957+561$ put new limits on the amplitude of low-frequency gravitational waves $h<2 \times 10^{-5}\left[\mathrm{v} /\left(3 \times 10^{-18} \mathrm{~Hz}\right)\right]$ in the frequency range $3 \times 10^{-18}$ $\mathrm{Hz}<v$. Future measurements of the time delay in other gravitational lenses may permit this limit to be improved.
\end{abstract}

PACS numbers: $04.80 .+\mathrm{z}, 98.60 . \mathrm{Vx}, 98.80 . \mathrm{Cq}$

Gravitational waves (GW's) have been detected indirectly through measurements of the rate at which the binary pulsar PSR $1913+16$ is speeding up. The development of laboratory-scale interferometric and bar antennae is proceeding at a rapid pace, and reliable $\mathrm{GW}$ detectors may be only a few years away. The construction of large-scale GW antennae in space has also been seriously proposed.

The different sources of GW's can be classified as being transient, periodic, or stochastic. The transient sources are those such as supernova explosions in which the GW's are produced by a single isolated event. The periodic sources are rapidly orbiting systems like the binary pulsar, which radiate GW's steadily over a long period of time. The stochastic sources are events which took place during the early history of the Universe, and which produce a background of random gravitational fluctuations. A period of exponential inflation in the early Universe ${ }^{1}$ and the gradual decay of a network of cosmic strings ${ }^{2}$ are two examples of such stochastic sources.

This paper shows how gravitational lenses ${ }^{3}$ can serve as moderately sensitive detectors of the long-wavelength part of this stochastic background. The sensitivity of this type of detector is greatest at wavelengths comparable to the horizon scale today, a range of frequency which is inaccessible to laboratory or space-based instruments. This low-frequency spectrum is of great interest because an inflationary epoch in the history of the Universe would leave its mark here. ${ }^{1}$

It is easy to see how a gravitational lens can serve as a $\mathrm{GW}$ detector. The source is at a cosmological distance, and its light reaches the Earth along two separated geodesics. Because of their enormous spatial length, these two geodesics have a maximum spatial separation of about $10^{23} \mathrm{~cm}$. Thus, they traverse different regions of space-time. Now imagine that a $\mathrm{GW}$ is present. This GW may be thought of as providing a small redshift along the path of one light ray, and a small blueshift along the other path. The differential redshift produces a time delay between the images, and thus the measured time delay can be used to place limits on the amplitude of the GW.

Of course, there is also an intrinsic time delay between the two paths, which is due to the curvature of spacetime and the geometry of the lens. Thus, it is possible (but improbable) that the intrinsic time delay is very large, but is almost completely canceled by the effect of a large-amplitude GW. In this paper, we make the reasonable assumption that the intrinsic geometrical delay is not much larger than the measured time delay. This assumption can be tested in the future, when the time delay of other gravitational lenses has also been determined. It may be possible to remove the assumption completely if one can measure the time delays in a gravitational lens with more than two images.

One may think of the gravitational lens as a crude sort of interferrometric GW antenna, of the type being developed for laboratory experiments. In the laboratory instruments, the two beams of light travel along perpendicular paths to maximize the sensitivity of the instrument. In the gravitational lens the sensitivity is decreased because the light rays do not follow perpendicular paths. However, the enormous length scales make it a useful detector just the same.

We begin by considering the time delay between the images of the gravitational lens in the presence of a plane GW. For simplicity we consider a system in flat space rather than in an expanding universe. Because the sources are at redshifts of order unity, this will give the correct order of magnitude.

The redshift $Z$ resulting from the GW along a given path is given by the formula of Sachs and Wolfe, ${ }^{4}$

$$
1+Z=1+\frac{1}{2} \int_{t_{o}}^{t_{e}} d t \dot{h}_{i j} e^{i} e^{j},
$$

where $t_{o}$ and $t_{e}$ are the times of observation and emission of the photon, $e^{i}$ is a spatial vector pointing from the observer to the source along the geodesic, the dot indicates a derivative with respect to time, and the integral is taken along the null geodesic followed by the photon. The $\mathrm{GW}$ is in a transverse traceless synchronous gauge, so that $h_{0 i}=h_{00}=h_{\mu v}{ }^{i v}=0$.

We suppose that the source is at the origin, and that 
the observer is on the $z$ axis. For a given wave vector $\mathbf{k}$, the gravitational metric perturbation can be expressed as the real part of

$h_{i j}=\left[\left(u_{i} u_{j}-v_{i} v_{j}\right) h_{+}+\left(u_{i} v_{j}+v_{i} u_{j}\right) h_{\times}\right] e^{i \omega t-i \mathbf{k} \cdot \mathbf{x}+i \delta}$,

where $h+$ and $h_{\times}$are the amplitudes of the two different polarizations, $\omega=|\mathbf{k}|$, $\mathbf{x}$ denotes the vector $(x, y, z)$, and $\delta$ is a real phase. The unit spatial vectors $\mathbf{u}$ and $\mathbf{v}$ are defined by

$$
\begin{aligned}
& u^{a}=\epsilon^{a b c} k_{b} z_{c} /\left[k_{d} k^{d}-\left(k_{d} z^{d}\right)^{2}\right]^{1 / 2}, \\
& v^{a}=\epsilon^{a b c} k_{b} u_{c} /\left(k_{d} k^{d}\right)^{1 / 2} .
\end{aligned}
$$

Here $\epsilon^{a b c}$ is the antisymmetric unit tensor, and $\mathbf{z}$ is a unit vector in the $z$ direction.

The previous expression for a gravitational perturbation is a standard one, usually written for a GW propagating in the $z$ direction. In our case, the GW is traveling in the $\hat{\mathbf{k}}$ direction, and we have written the usual expression with respect to a new orthogonal triad of vectors $(\hat{\mathbf{u}}, \hat{\mathbf{v}}, \hat{\mathbf{k}})$.

For simplicity, we approximate the two image paths by the four straight-line segments shown in Fig. 1. These line segments lie in the plane $y=0$ and have length $L$. The unit vectors pointing along the two directions to the images are denoted $\hat{\mathbf{m}}_{1}$ and $\hat{\mathbf{m}}_{2}$, and the angular separation between the two images is $2 \eta$. The wave vector $\mathbf{k}$ is given in spherical polar coordinates by the two angles $\theta$ and $\phi$. The $(x, y, z)$ components of the various vectors are then

$$
\begin{aligned}
& \mathbf{k}=\omega(\sin \theta \cos \phi, \sin \theta \sin \phi, \cos \theta) \\
& \hat{\mathbf{u}}=(\sin \phi,-\cos \phi, 0) \\
& \hat{\mathbf{v}}=(\cos \theta \cos \phi, \cos \theta \sin \phi,-\sin \theta), \\
& \hat{\mathbf{m}}_{1}=(-\sin \eta, 0, \cos \eta), \quad \hat{\mathbf{m}}_{2}=(\sin \eta, 0, \cos \eta)
\end{aligned}
$$

The GW creates a difference between the redshifts

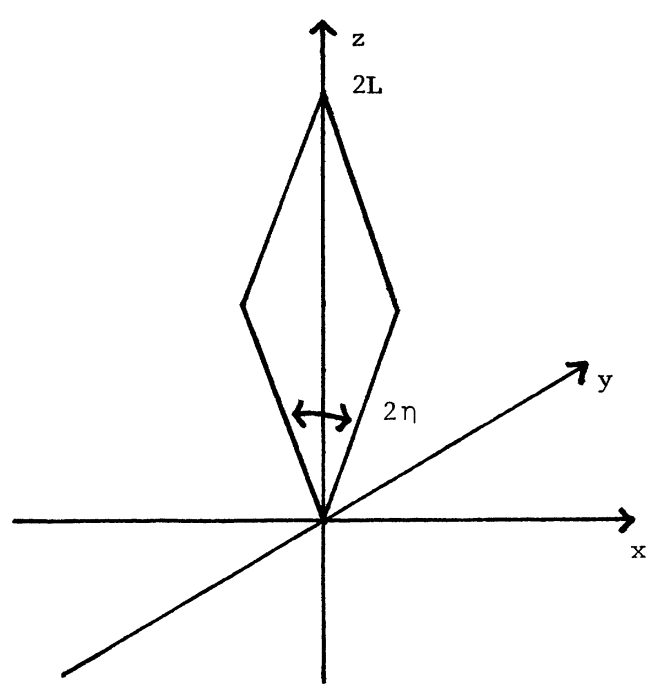

FIG. 1. The path taken by two light rays in a simple model of a gravitational lens. The small angular separation between the two rays is $2 \eta$.

along the two paths, which is given by the real part of

$$
\Delta Z=\frac{1}{2} i \omega\left(\int_{\text {path } 1} h_{i j} e^{i} e^{j} d t-\int_{\text {path } 2} h_{i j} e^{i} e^{j} d t\right) .
$$

These integrals can now be evaluated exactly. One obtains the real part of

$$
\begin{aligned}
\Delta Z= & \frac{1}{2} \omega e^{i \delta+i \omega t_{e}}\left[\frac{S_{i j} m_{1}^{i} m_{1}^{j}}{i\left(\omega-k_{i} m_{1}^{i}\right)}-\frac{S_{i j} m_{2}^{i} m_{2}^{j}}{i\left(\omega-k_{i} m_{2}^{i}\right)}\right] \\
& \times\left(e^{i\left(\omega-k_{i} m_{1}^{i}\right) L}-1\right)\left(e^{i\left(\omega-k_{i} m_{2}^{i}\right) L}-1\right),
\end{aligned}
$$

where

$$
S_{i j}=\left(u_{i} u_{j}-v_{i} v_{j}\right) h_{+}+\left(u_{i} v_{j}+v_{i} u_{j}\right) h_{\times} .
$$

The quantity in square brackets is an odd function of $\eta$; the remaining part of the expression is even in $\eta$. The dot product between $S_{i j}$ and $\hat{\mathbf{m}}_{1}$ is given by

$$
S_{i j} m_{1}^{i} m_{1}^{j}=h_{+}\left[\sin ^{2} \eta \sin ^{2} \phi-(\sin \eta \cos \theta \cos \phi+\cos \eta \sin \theta)^{2}\right]+2 h_{\times} \sin \eta \sin \phi(\sin \eta \cos \theta \cos \phi+\cos \eta \sin \theta),
$$

and the dot product $S_{i j} m_{2}^{i} m_{2}^{j}$ is the same except that the sign of $\eta$ is reversed. Expanding $\Delta Z$ for small values of $\eta$, one obtains the real part of

$$
\Delta Z=4 \eta e^{i \delta+i \omega t_{e}+i \omega L(1-\cos \theta)} \sin ^{2}\left(\frac{\omega L}{2}(1-\cos \theta)\right) \sin \theta\left(\cos \phi h_{+}+\frac{2 \sin \phi}{1-\cos \theta} h_{\times}\right) .
$$

For a given fixed separation between the source and observer, and a fixed plane $\mathrm{GW}$, the difference in redshift oscillates periodically in time $t_{e}=t_{o}-2 L$, with the period $\omega$ of the GW.

The connection between the differential redshift $\Delta Z$ and the time delay between the two images is easily obtained. Imagine that a series of pulses are emitted by the source at regular intervals $d t_{e}$. These pulses arrive at the observer at regular intervals $\left(d t_{o}\right)_{1}$ along the first path and $\left(d t_{o}\right)_{2}$ along the second path. The time delay between successive pulses is related to the difference in redshift by

$$
\Delta Z=\left(d t_{o}\right)_{1} / d t_{e}-\left(d t_{o}\right)_{2} / d t_{e} .
$$

Thus the total time delay between the images seen via the two paths can accumulate over the period of the 
GW:

$$
\Delta T=\left(t_{o}\right)_{1}-\left(t_{o}\right)_{2}=\int d t_{e} \Delta Z .
$$

Evaluating this integral, one obtains for the time delay between the two paths the real part of

$\Delta T=\Delta Z / i \omega+\Delta T_{\text {intrinsic }}$, where $\Delta T_{\text {intrinsic }}$ is the constant of integration. As discussed earlier, we assume that the intrinsic geometrical part of the time delay is not much larger than the measured time delay, so we can neglect $\Delta T_{\text {intrinsic. The di- }}$ mensionless fractional time delay $\Delta T / T$ is then obtained by dividing this formula by $T=2 L$, which is the total light travel time from the source to the observer:

$$
\frac{\Delta T}{T}=\frac{4 \eta}{\omega T} \cos \left(\delta+\omega t_{e}+\omega L(1-\cos \theta)-\frac{\pi}{2}\right) \sin ^{2}\left(\frac{\omega T}{4}(1-\cos \theta)\right) \sin \theta\left(\cos \phi h_{+}+\frac{2 \sin \phi}{1-\cos \theta} h_{\times}\right) .
$$

We now assume that the background of gravitational radiation at a given wavelength is isotropic, i.e., that the amplitude of the radiation as a function of $\mathbf{k}$ depends only upon the length of $\mathbf{k}$ and not upon its direction. The phases $\delta$ for the different values of $\mathbf{k}$ are assumed to be independent random variables, uniformly distributed between 0 and $2 \pi$. The amplitudes $h_{+}$and $h_{\times}$for different values of $\mathbf{k}$ are assumed to be independent random variables, with vanishing mean values. The mean values of their squares are taken to be zero except for wave vectors $\mathbf{k}$ of a given fixed length, where the mean values of the squares are taken to be a constant, independent of the directions $\theta$ and $\phi$. The mean value of $h_{i j} h^{i j}$ is then given by

$$
\left\langle h^{i j} h_{i j}\right\rangle=\left\langle h_{+}^{2}\right\rangle+\left\langle h_{\times}^{2}\right\rangle=h^{2},
$$

which serves to define the dimensionless gravitational strain amplitude $h$.

The quantity $(\Delta T / T)^{2}$ can now be averaged over phase $\delta$ and over $4 \pi$ solid angle in the direction of $\mathbf{k}$ to obtain a mean-square value:

$$
\left\langle(\Delta T / T)^{2}\right\rangle=\int_{0}^{2 \pi} \frac{d \delta}{2 \pi} \int_{0}^{2 \pi} \frac{d \phi}{2 \pi} \int_{0}^{\pi} \frac{\sin \theta}{2} d \theta\left(\frac{\Delta T}{T}\right)^{2} .
$$

In the averaging over the phase $\delta$, the first cosinesquared term appearing in the formula for $(\Delta T / T)^{2}$ averages to $\frac{1}{2}$ and so the dependence on the time $t_{e}$ drops out. The average over $\phi$ also gives a factor of $\frac{1}{2}$. Thus setting $s=1-\cos \theta$ one obtains

$$
\begin{aligned}
&\langle\Delta T / T\rangle_{\mathrm{rms}}=\left\langle(\Delta T / T)^{2}\right\rangle^{1 / 2} \\
&=\left[\frac { \eta } { \omega T } \left[\int_{0}^{2} \sin ^{4}\left(\frac{\omega T s}{4}\left(4 s-2 s^{2}\right)\right)\right.\right. \\
&\left.\times\left(\left\langle h_{+}^{2}\right\rangle+\frac{4}{s^{2}}\left\langle h_{\times}^{2}\right\rangle\right) d s\right]^{1 / 2} .
\end{aligned}
$$

If we assume that the gravitational background radiation is unpolarized, then $\left\langle h_{+}^{2}\right\rangle=\left\langle h_{\times}^{2}\right\rangle=h^{2} / 2$. One then obtains an rms time delay of the form

$$
\langle\Delta T / T\rangle_{\mathrm{rms}}=\eta h f(\omega T),
$$

where the frequency response function $f(x)$ is given by

$$
f(x)=x^{-1}\left[\int_{0}^{2} d s \sin ^{4}[x s / 4](2-s)(s+4 / s)\right]^{1 / 2} .
$$

The function $f(x)$ vanishes for small $x<1$ like $f(x) \propto x$. Thus the detector is not sensitive to wavelengths longer than the overall size of the gravitational lens. For large $x>1$ it falls off like $f(x) \propto(\ln x)^{1 / 2} / x$. This means that the detector also loses sensitivity at short wavelengths. The maximum value of $f$ is $f(3.7) \approx 0.41$, and a typical value is $f(2 \pi) \approx 0.33$. The detector is thus most sensitive to wavelengths of the same characteristic size as the gravitational lens.

Thus the rms fractional time delay due to GW's of frequency $\omega$ and amplitude $h$ is given by

$$
\langle\Delta T / T\rangle_{\mathrm{rms}} \approx 2 \eta h / \omega T \text {. }
$$

This approximation is good provided that $\omega T>\pi$. The sensitivity of the detector is thus reduced by a factor of $\eta$ from the sensitivity of a detector with perpendicular arms.

The measured time delay of the gravitational lens $0957+561$ is $415 \pm 20$ days. ${ }^{5}$ The light takes about $10^{10}$ years to reach us, and thus $\Delta T / T<10^{-10}$. The angular scale of separation of the two observed images is $2 \eta=6.1$ $\operatorname{arcsec}=3 \times 10^{-5}$ rad. Hence one obtains limits on the GW amplitude in the frequency range

$$
3 \times 10^{-18} \mathrm{~Hz}<v,
$$

where the angular frequency $\omega=2 \pi v$. In this frequency range the dimensionless amplitude $h$ is less than

$$
h<2 \times 10^{-5}\left(\frac{v}{3 \times 10^{-18} \mathrm{~Hz}}\right) .
$$

We have dropped the logarithmic term from this formula, as it only changes the result by a small factor.

The only existing limits in this frequency range come from the limits on the temperature fluctuations of the microwave background radiation, ${ }^{6}$ which give $h<10^{-6}$ in the frequency range $10^{-16} \mathrm{~Hz}<v<10^{-14} \mathrm{~Hz}$, and $h<3 \times 10^{-4}$ in the frequency range $10^{-18} \mathrm{~Hz}<v$ $<10^{-16} \mathrm{~Hz}$.

The improved bounds that we have given at lower frequencies can be used to put new limits on the energy density during axn inflationary stage of expansion in the early Universe. Because the low-frequency amplitude of the gravitational radiation scales as the fourth power of the grand-unification scale $M_{x},{ }^{1}$ the existing bound ${ }^{7}$ on 
$M_{x}$ is improved by a factor of $10^{0.25}$. One thus obtains $M_{x}<5 \times 10^{16} \mathrm{GeV}$ in the inflationary-universe models. If gravitational lenses with smaller time delays and larger angular separations are found, these bounds could be further improved.

I would like to thank L. Ford, J. Friedmann, W. Hiscock, H. Jones, Jr., L. Parker, K. Thorne, E. Turner, and R. Weiss for useful discussions. I am especially grateful to $\mathrm{W}$. Hiscock for pointing out a serious error in an earlier version of this work. This work was partially supported by NSF Grants No. PHY87-05107 and No. PHY 89-03027.

${ }^{1}$ B. Allen, Phys. Rev. D 37, 2078 (1988); M. Ted Ressell and Michael S. Turner, Fermilab Report No. Fermilab-PUB89/179-A, 1989 (to be published).
${ }^{2}$ R. Brandenburger, A. Albrecht, and N. Turok, Nucl. Phys. B277, 605 (1986); J. Traschen, N. Turok, and R. Brandenburger, Phys. Rev. D 34, 919 (1986).

${ }^{3}$ Edwin L. Turner, in Proceedings of the Fourteenth Texas Symposium on Relativistic Astrophysics, edited by E. J. Fenyves (New York Academy of Science, New York, 1989); in Gravitational Lenses, edited by J. M. Moran, J. N. Hewitt, and K. Y. Lo, Lecture Notes in Physics Vol. 330 (SpringerVerlag, Berlin, 1989), p. 69.

${ }^{4}$ R. Sachs and A. Wolfe, Astrophys. J. 147, 173 (1967).

${ }^{5}$ C. Vanderriest, J. Schneider, G. Herpe, M. Chevreton, M. Moles, and G. Wlérick, Astron. Astrophys. 215, 1 (1989).

${ }^{6} \mathrm{D}$. T. Wilkinson, in Proceedings of the Thirteenth Texas Symposium on Relativistic Astrophysics, edited by M. Ulmer (World Scientific, Singapore, 1987), p. 209.

${ }^{7}$ A. Starobinsky, Pis'ma Zh. Eksp. Teor. Fiz. 30, 719 (1979) [JETP Lett. 30, 682 (1979)]; V. A. Rubakov, M. V. Sazhin, and A. V. Veryaskin, Phys. Lett. 115B, 189 (1982); R. Fabbri and M. Pollock, Phys. Lett. 125B, 445 (1983). 